\title{
Domination topological properties of polyhydroxybutyrate and polycaprolactone with QSPR analysis
}

\author{
Hanan Ahmed ${ }^{1}$, Anwar Alwardi ${ }^{2}$, Suha A. Wazzan ${ }^{3}$ \\ ${ }^{1}$ Department of Mathematics, Yuvarajas College, University of Mysore, Mysore, India \\ ${ }^{2}$ Department of Mathematics, University of Aden, Yemen \\ ${ }^{3}$ Department of Mathematics, Science Faculty, King Abdulaziz University, Jeddah, Saudi Arabia \\ hananahmed1a@gmail.com, a_wardi@hotmail.com,swazzan@kau.edu.sa
}

DOI 10.17586/2220-8054-2021-12-6-664-671

\begin{abstract}
Polyhydroxyalkanoates (PHAs) are biopolymers, which are stored inside cells as energy storage materials by various microorganisms. PHAs are plastic materials that have a positive environmental impact when compared to regular plastics in terms of production methods and recyclabillity. In addition, PHAs are characterized by biocompatibility, biodegradability and thus have a wide range of applications such as biomedicine, surgery, etc. The most common PHAs are Polyhydroxybutyrate, Polyhydroxyvalerate, and copolymer. In this paper, we calculate the domination topological indices of these polymers; also, we discuss the quantitative structure property relationships (QSPR) analysis of these domination topological indices. Further, we show that the characteristics have a good correlation with the physico-chemical characteristics of polymers.
\end{abstract}

Keywords: polyhydroxybutyrate, polyhydroxyvalerate, domination topological indices, domination degree, QSPR analysis.

Received: 18 November 2021

Revised: 27 November 2021

Final revision: 29 November 2021

\section{Introduction}

Polyhydroxyalkanoates (PHAs) are biologically produced in various biological organisms. There are several microorganisms known as the gatherer of PHAs such as Pseudomonas sp., Bacillus sp., etc [1]. Where the PHAs are stored and mixed as granules in the cytoplasm [2]. PHAs have a wide range of applications such as biomedicine [3,4]. PHAs are similar in terms of their physical and chemical properties to oil-based plastics such as polypropylene [5-7]. Let $G=(V, E)$ be a connected, simple graph with vertex set $V$ and edge set $E$. A set $D \subseteq V$ is said to be a dominating set of a graph $G$, if for any vertex $v \in V-D$, there exists a vertex $u \in D$ such that $u$ and $v$ are adjacent. The domination number $\gamma(G)$ of a graph $G$ is the minimum cardinality of a minimal dominating set in $G$. A dominating set $D=\left\{v_{1}, v_{2}, \ldots, v_{r}\right\}$ is minimal if $D-v_{i}$ is not a dominating set [8]. A dominating set of $G$ of minimum cardinality is said to be a minimum dominating set. Topological indices are the numerical parameters of a graph, and these parameters are the same for graphs which are isomorphic. A variety of topological indices have been created and developed, and many studies have been conducted on them in various fields of molecular graphs and networks [9-14]. A. M. Hanan Ahmed et al. [15], have introduced new degree-based topological indices called domination topological indices which are based on the minimal dominating sets. The domination degree is defined as:

Definition 1.1. [15] For each vertex $v \in V(G)$, the domination degree denotes by $d_{d}(v)$ and define as the number of minimal dominating sets of $G$ which contains $v$.

The first and second domination Zagreb indices and modified first Zagreb domination indices are defined as:

$$
\begin{gathered}
D M_{1}(G)=\sum_{v \in V(G)} d_{d}^{2}(v), \\
D M_{2}(G)=\sum_{u v \in E(G)} d_{d}(u) d_{d}(v), \\
D M_{1}^{*}(G)=\sum_{u v \in E(G)}\left[d_{d}(u)+d_{d}(v)\right],
\end{gathered}
$$

where $d_{d}(v)$ is the domination degree of the vertex $v$. The total number of minimal dominating sets of $G$ is denoted as $T_{m}(G)$ [15]. The forgotten domination, hyper domination, and modified forgotten domination indices of graphs [16] are defined as:

$$
D F(G)=\sum_{v \in V(G)} d_{d}^{3}(v),
$$




$$
\begin{aligned}
& D H(G)=\sum_{u v \in E(G)}\left[d_{d}(u)+d_{d}(v)\right]^{2}, \\
& D F^{*}(G)=\sum_{u v \in E(G)} d_{d}^{2}(u)+d_{d}^{2}(v) .
\end{aligned}
$$

For more discussion about domination topological indices, refer to [10-12].

\section{Materials and methods}

In this paper, the results will be organized into two parts:the first part, in which the total number of minimal dominating sets are determined, and then the domination degree for all vertices is calculated. Using the domination degree, the exact values of the domination topological indices are calculated. In the second part, the quantitative structure property relationships (QSPR) analysis of these indicators is discussed, as well as the verification of the chemical applicability of the domination topological indicators. A set of physical and chemical properties of polymers were considered for such a test and the corresponding experimental values are given in Table 1.Analysis tools the linear structures of the models obtained by the program are drawn Excel. For the nonlinear regression analysis, we use R-software.

TABLE 1. Physiochemical properties of PHB, PHV, and PHBV such as Melting Point (M.P.)

\begin{tabular}{|c|c|}
\hline Polymer name & M.P. $C^{\circ}$ \\
\hline \hline PHB[n] & 170 \\
\hline PHV[n] & 180 \\
\hline PHBV[n] & 145 \\
\hline
\end{tabular}

\section{Main results}

Polyhydroxybutyrate (PHB) is one type of PHA and, it is of great importance, as biologically derived plastics have the potential for biodegradability [17]. In this section, we calculate the domination topological indices of degradable plastics such as PHB. We will use the symbol PHB[n] (see Fig. 1) for one layer of this structure containing $n$ connections together. The substance chart PHB[n] contains $12 n$ vertices and $12 n-1$ edges, where $n$ is the quantity of connections in a layer.

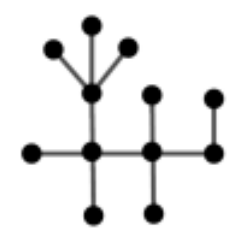

(a)

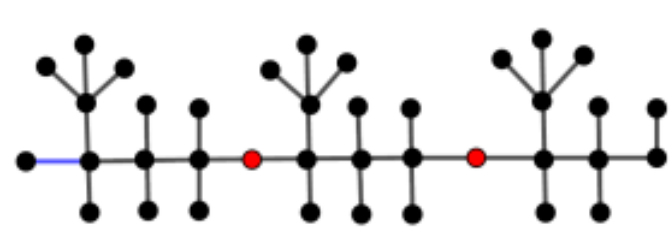

(b)

FIG. 1. Polyhydroxybutyrate PHB[n], (a) unit of PHB[n], (b) appear PHB[3]

Lemma 3.1. Let $G \cong P H B[n]$, for $n \geq 1$. Then $T_{m}(G)=16 n$, and

$$
d_{d}(v) \begin{cases}2^{4 n-2}, & \text { if } v \text { is the common vertex } \\ 2^{4 n-1}, & \text { otherwise. }\end{cases}
$$

Proof. Let $G$ be the molecular graph of Polyhydroxybutyrate PHB[n]. We first divide $G$ into $n$ components $A_{1}, A_{2}$, $A_{3}, \ldots, A_{n}$. We calculate the minimal dominating sets of each component so that we get, $T_{m}\left(A_{1}\right)=16, T_{m}\left(A_{2}\right)=16$, $T_{m}\left(A_{3}\right)=16, \ldots, T_{m}\left(A_{n}\right)=16$. Every minimal dominating set of $A_{1}$ is added to each minimal dominating set of $A_{2}$ and we check for the minimality of the resulting dominating sets. As a result, we obtain 256 minimal dominating sets. Note that the common vertex will be removed if this vertex is present in the same minimal dominating set with that vertex in the first unit which is adjacent to it. 
Next, every minimal dominating set of $A_{3}$ is added to each of these 256 minimal dominating sets and we check for the minimality of the resulting dominating sets. Similarly, the second common vertex will be removed if this vertex is present in the same minimal dominating set with that vertex in the previous unit which is adjacent to it. Here, we obtain $256 \times 16=4096$ minimal dominating sets. Continuing in this manner we get $T_{m}(G)=16 n$, and

$$
d_{d}(v)= \begin{cases}2^{4 n-2}, & \text { if } v \text { is the common vertex } \\ 2^{4 n-1}, & \text { otherwise. }\end{cases}
$$

Theorem 3.2. Suppose $G$ is the molecular graph of Polyhydroxybutyrate $\operatorname{PHB}[\mathrm{n}]$, for $n \geq 1$. Then:

$$
\begin{gathered}
D M_{1}(G)=2^{8 n-4}(45 n+3), \\
D M_{2}(G)=2^{8 n-2}(10 n+1)+2^{8 n-3}(2 n-2), \\
D M_{1}^{*}(G)=2^{4 n}(10 n+1)+\left(2^{4 n-1}+2^{4 n-2}\right)(n-1) .
\end{gathered}
$$

Proof. if $G \cong P H B[n]$, the set of vertices of $G$ divides into two sets, $C$ is the set of all common vertices, $|C|=n-1$ and $B$ contains another vertex of $G,|B|=11 n+1$. By using Lemma 3.1, we have:

$$
D M_{1}(G)=\sum_{v \in V(G)} d_{d}^{2}(v)=\sum_{v \in V(B)}\left(\left(2^{4 n-1}\right)^{2}+\sum_{v \in V(C)}\left(2^{4 n-2}\right)^{2}=2^{8 n-4}(45 n+3) .\right.
$$

From Table 2, we get:

$$
\begin{gathered}
D M_{2}(G)=\sum_{u v \in E(G)} d_{d}(u) d_{d}(v)=2^{8 n-2}(10 n+1)+2^{8 n-3}(2 n-2), \\
D M_{1}^{*}(G)=\sum_{u v \in E(G)} d_{d}(u)+d_{d}(v)=2 \times 2^{4 n-1}+\left(2^{4 n-1}+2^{4 n-2}\right)(2 n-2)+2 \times 2^{4 n-1}(10 n) \\
=2^{4 n}(10 n+1)+\left(2^{4 n-1}+2^{4 n-2}\right)(n-1) .
\end{gathered}
$$

The edges of $G$ are separated as follows (Table 2).

TABLE 2. Edge partition of PHB[n]

\begin{tabular}{|c|c|}
\hline$\left(d_{d}(u), d_{d}(v)\right)$ & Number of edges \\
\hline \hline$\left(2^{4 n-1}, 2^{4 n-1}\right)$ & 1 (First edge in first unit) \\
\hline$\left(2^{4 n-1}, 2^{4 n-2}\right)$ & $2 n-2$ \\
\hline$\left(2^{4 n-1}, 2^{4 n-1}\right)$ & $10 n$ \\
\hline
\end{tabular}

Theorem 3.3. If $G \cong \mathrm{PHB}[\mathrm{n}]$, for $n \geq 1$, then:

$$
\begin{gathered}
D F(G)=2^{12 n-6}(89 n+7), \\
D H(G)=2^{8 n}(10 n+1)+\left(2^{8 n}+2^{8 n-3}\right)(n-1) \\
D F^{*}(G)=2^{8 n-1}(10 n+1)+\left(2^{8 n-1}+2^{8 n-3}\right)(n-1) .
\end{gathered}
$$

Proof. Let $G \cong \operatorname{PHB}[\mathrm{n}]$, for $n \geq 1$. By using the partition of vertices of $G$ as in proof of Theorem 3.2, and Lemma 3.1, we get:

$$
\begin{aligned}
D F(G)=\sum_{v \in V(G)} d_{d}^{3}(v)=\sum_{v \in V(B)}\left(\left(2^{4 n-1}\right)^{3}\right. & +\sum_{v \in V(C)}\left(2^{4 n-2}\right)^{3} \\
& =\left[\frac{88 \times 2^{12 n-6}+2^{12 n-6}}{64}\right] n+7 \times 2^{12 n-6}=2^{12 n-6}(89 n+7) .
\end{aligned}
$$

From Table 2, we have:

$$
\begin{aligned}
D H(G) & =\sum_{u v \in E(G)}\left(d_{d}(u)+d_{d}(v)\right)^{2}=2^{8 n}(10 n+1)+\left(2^{8 n}+2^{8 n-3}\right)(n-1), \\
D F^{*}(G) & =\sum_{u v \in E(G)} d_{d}^{2}(u)+d_{d}^{2}(v)=2^{8 n-1}(10 n+1)+\left(2^{8 n-1}+2^{8 n-3}\right)(n-1) .
\end{aligned}
$$


Now, we compute domination topological indices of Polyhydroxyvalerate (PHV) biodegradable plastic, we use the notation PHV[n] (see Fig. 2) for one layer of this structure contains $n$ unites. The chemical structure of PHV[n] consists of $15 n$ vertices and $15 n-1$ edges, where $n$ is the number of units in a layer.

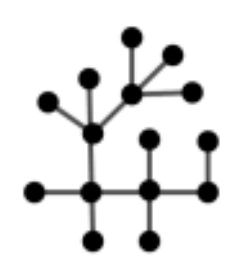

(a)

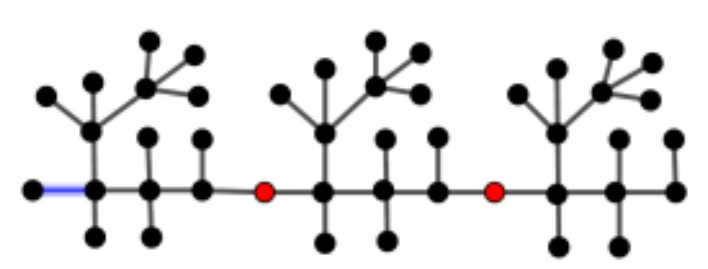

(b)

FIG. 2. Polyhydroxyvalerate PHV[n], (a) unit of PHV[n], (b) represents PHV[3]

Lemma 3.4. Let $G \cong \mathrm{PHV}[\mathrm{n}]$, for $n \geq 1$. Then $T_{m}(G)=32 n$ and:

$$
d_{d}(v)= \begin{cases}2^{5 n-2}, & \text { if } v \text { is the center vertex } \\ 2^{5 n-1}, & \text { otherwise. }\end{cases}
$$

Proof. The proof of this lemma is on the same line as that of Lemma 3.1.

Theorem 3.5. Let $G$ be the chemical structure of PHV[n], for $n \geq 1$. Then:

$$
\begin{gathered}
D M_{1}(G)=\left(7 \times 2^{10 n-1}+2^{10 n-4}\right) n+3 \times 2^{10 n-4}, \\
D M_{2}(G)=7 \times 2^{10 n-1} n \\
D M_{1}^{*}(G)=2^{5 n}(13 n+1)+3 \times 2^{5 n-1}(n-1) .
\end{gathered}
$$

Proof. Let $G \cong \operatorname{PHV}[\mathrm{n}]$, we can divide the vertices of $G$ into two sets: the set $C$ which contains the common vertices, $|C|=n-1$ and the set $B$, which contains the other vertices of $G,|B|=14 n+1$ by using Lemma 3.4, we get:

$$
\begin{aligned}
D M_{1}(G)= & \sum_{v \in V(G)} d_{d}^{2}(v)=\sum_{v \in V(B)}\left(\left(2^{5 n-1}\right)^{2}+\sum_{v \in V(C)}\left(2^{5 n-2}\right)^{2}\right. \\
& =\left(\frac{7 \times 2^{10 n+3}+2^{10 n}}{16}\right) n+4 \times 2^{10 n-4}-2^{10 n-4}=\left(7 \times 2^{10 n-1}+2^{10 n-4}\right) n+3 \times 2^{10 n-4}
\end{aligned}
$$

From Table 3, we get:

$$
\begin{gathered}
D M_{2}(G)=\sum_{u v \in E(G)} d_{d}(u) d_{d}(v)=7 \times 2^{10 n-1} n, \\
D M_{1}^{*}(G)=\sum_{u v \in E(G)} d_{d}(u)+d_{d}(v)=2^{5 n}(13 n+1)+3 \times 2^{5 n-1}(n-1) .
\end{gathered}
$$

The edges of $G$ are separated as follows (Table 3).

TABLE 3. Edge partition of PHV[n]

\begin{tabular}{|c|c|}
\hline$\left(d_{d}(u), d_{d}(v)\right)$ & Number of edges \\
\hline \hline$\left(2^{5 n-1}, 2^{5 n-1}\right)$ & 1 (first edge in the first unit) \\
\hline$\left(2^{5 n-1}, 2^{5 n-2}\right)$ & $2 n-2$ \\
\hline$\left(2^{5 n-1}, 2^{5 n-1}\right)$ & $13 n$ \\
\hline
\end{tabular}

Theorem 3.6. Suppose $G$ is the molecular structure of PHV[n], then:

$$
\begin{gathered}
D F(G)=\left(7 \times 2^{15 n-2}+2^{15 n-6}\right) n+7 \times 2^{15 n-6}, \\
D H(G)=2^{10 n}(13 n+1)+\left(2^{10 n}+2^{10 n-3}\right)(n-1), \\
D F^{*}(G)=2^{10 n-1}(13 n+1)+2^{10 n-3}(5 n-5) .
\end{gathered}
$$


Proof. Let $G \cong \mathrm{PHV}[\mathrm{n}]$, for $n \geq 1$. By using the partition of vertices of $G$ as in the proof of Theorem 3.5 and Lemma 3.4, we get:

$$
\begin{aligned}
D F(G)=\sum_{v \in V(G)} d_{d}^{3}(v) & =\sum_{v \in V(B)}\left(\left(2^{5 n-1}\right)^{3}+\sum_{v \in V(C)}\left(2^{5 n-2}\right)^{3}\right. \\
& =\left(\frac{7 \times 2^{15 n+4}+2^{15 n}}{64}\right) n+7 \times 2^{15 n-6}=\left(7 \times 2^{15 n-2}+2^{15 n-6}\right) n+7 \times 2^{15 n-6} .
\end{aligned}
$$

From Table 3, we have:

$$
\begin{gathered}
D H(G)=\sum_{u v \in E(G)}\left(d_{d}(u)+d_{d}(v)\right)^{2}=2^{10 n}(13 n+1)+\left(2^{10 n}+2^{10 n-3}\right)(n-1), \\
D F^{*}(G)=\sum_{u v \in E(G)} d_{d}^{2}(u)+d_{d}^{2}(v)=2^{10 n-1}(13 n+1)+2^{10 n-3}(5 n-5) .
\end{gathered}
$$

In this part we shall compute domination topological indices of chemical structure of the co-polymer of PHB and PHV. One layer of this structure we denote it by PHBV[n] (see Fig. 3) containing $n$ units. The chemical graph PHBV[n] contains $27 n$ vertices and $27 n-1$ edges.

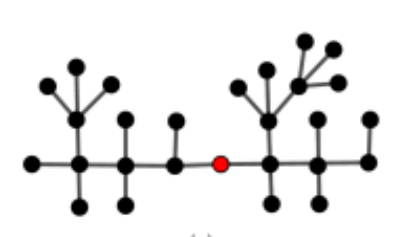

(a)

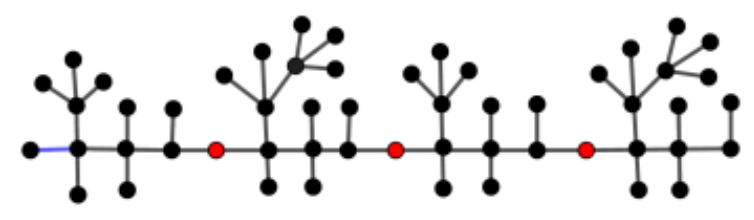

(b)

FIG. 3. Polyhydroxybutyrovalerate for the co-polymer PHBV[n], (a) unit of PHBV[n], (b) appear PHBV[2]

Lemma 3.7. Let $G \cong \operatorname{PHBV[n],~for~} n \geq 1$. Then $T_{m}(G)=512 n$ and

$$
d_{d}(v)= \begin{cases}2^{9 n-2}, & \text { if } v \text { is the center vertex } \\ 2^{9 n-1}, & \text { otherwise. }\end{cases}
$$

Proof. The proof of this lemma is on the same line as that of Lemma 3.1.

Theorem 3.8. Suppose $G$ is the molecular structure of PHBV[n]. Then:

$$
\begin{gathered}
D M_{1}(G)=51 \times 2^{18 n-3} n+3 \times 2^{18 n-4}, \\
D M_{2}(G)=\left(23 \times 2^{18 n-2}+2^{18 n-1}\right) n, \\
D M_{1}^{*}(G)=2^{9 n}(23 n+4)-3 \times 2^{9 n-1} .
\end{gathered}
$$

Proof. Let $G \cong \mathrm{PHBV}[\mathrm{n}]$. The set of vertices of $G$ can be divided into two sets: the set $C$ contains all center vertices, $|C|=2 n-1$ and the set $B$ contains the remaining vertices of $G,|B|=25 n+1$. By using Lemma 3.7, we get:

$$
\begin{aligned}
D M_{1}(G)=\sum_{v \in V(G)} d_{d}^{2}(v)=\sum_{v \in V(B)} & \left(\left(2^{9 n-1}\right)^{2}+\sum_{v \in V(C)}\left(2^{9 n-2}\right)^{2}\right. \\
& =\left(\frac{50 \times 2^{18 n}+2^{18 n}}{8}\right) n+3 \times 2^{18 n-4}=51 \times 2^{18 n-3} n+3 \times 2^{18 n-4} .
\end{aligned}
$$

From Table 4, we have:

$$
\begin{aligned}
D M_{2}(G) & =\sum_{u v \in E(G)} d_{d}(u) d_{d}(v),=\left(23 \times 2^{18 n-2}+2^{18 n-1}\right) n \\
D M_{1}^{*}(G) & =\sum_{u v \in E(G)} d_{d}(u)+d_{d}(v)=2^{9 n}(23 n+4)-3 \times 2^{9 n-1} .
\end{aligned}
$$

The edges of $G$ are separated as follows (Table 4).

Theorem 3.9. Let $G \cong$ chemical structure of PHBV[n], for $n \geq 1$. Then:

$$
D F(G)=101 \times 2^{27 n-5} n+7 \times 2^{27 n-6},
$$


TABLE 4. Edge partition of PHBV[n]

\begin{tabular}{|c|c|}
\hline$\left(d_{d}(u), d_{d}(v)\right)$ & Number of edges \\
\hline \hline$\left(2^{9 n-1}, 2^{9 n-1}\right)$ & 1 (first edge in the first unit) \\
\hline$\left(2^{9 n-1}, 2^{9 n-2}\right)$ & $4 n-2$ \\
\hline$\left(2^{9 n-1}, 2^{9 n-1}\right)$ & $23 n$ \\
\hline
\end{tabular}

$$
\begin{aligned}
D H(G) & =2^{18 n}(23 n+1)+9\left(2^{18 n-2} n-2^{18 n-3}\right), \\
D F^{*}(G) & =2^{18 n-1}(23 n+1)+5\left(2^{18 n-2}-2^{18 n-3}\right) .
\end{aligned}
$$

Proof. Suppose $G \cong \operatorname{PHBV}[\mathrm{n}]$, for $n \geq 1$. By using the partition of vertices of $G$ as in the proof of Theorem 3.8 and Lemma 3.7, we get:

$$
D F(G)=\sum_{v \in V(G)} d_{d}^{3}(v)=\sum_{v \in V(B)}\left(\left(2^{9 n-1}\right)^{3}+\sum_{v \in V(C)}\left(2^{9 n-2}\right)^{3}=101 \times 2^{27 n-5} n+7 \times 2^{27 n-6} .\right.
$$

Now, from Table 4, we have:

$$
\begin{aligned}
& D H(G)=\sum_{u v \in E(G)}\left(d_{d}(u)+d_{d}(v)\right)^{2}=2^{18 n}(23 n+1)+9\left(2^{18 n-2} n-2^{18 n-3}\right), \\
& D F^{*}(G)=\sum_{u v \in E(G)} d_{d}^{2}(u)+d_{d}^{2}(v)=2^{18 n-1}(23 n+1)+5\left(2^{18 n-2}-2^{18 n-3}\right) .
\end{aligned}
$$

\section{QSPR Analysis}

QSPR analysis remains the focus of many studies aimed at the modeling and prediction of physicochemical and biological properties of molecules. A powerful tool to help in this task is chemometrics, which uses statistical and mathematical methods to extract maximum information from a data set. QSPR uses chemometric methods to describe how a given physicochemical property varies as a function of molecular descriptors relevant to the chemical structure of a molecule. Thus, it is possible to replace costly biological tests or experiments of a given physicochemical property with calculated descriptors, which can, in turn, be used to predict the properties of interest for new compounds. The basic strategy of QSPR is to find an optimum quantitative relationship, which can be used for the prediction of the properties of compounds, including those unmeasured. It is obvious that the performance of QSPR model mostly depends on the parameters used to describe the molecular structure. Many efforts have been made to develop alternative molecular descriptors which can be derived using only the information encoded in the chemical structure. Much attention has been concentrated on "topological indices" derived from the connectivity and composition of a molecule which has made significant contributions in QSPR studies. The topological index has advantages of simplicity and quick speed of computation and so attracts the attention of scientists.

In this section, we are going to discuss the QSPR analysis of the domination topological indices. Further, we show that the characteristics have a good correlation with the physico-chemical characteristics of polymers. In this part, we will show the importance of domination topological indices to predict the physiochemical property in Table 1 . In this study, we used the nonlinear regression analysis modelled as: $\log (y)=a+b \log (x)$, where $y$ is the physicochemical property of the chemical compounds and $x$ represents the domination topological indices. These were calculated using R-software for the values of one physicochemical property and the six domination topological indices of PHB, PHV, and Copolymer (PHBV) for $n=1, n=2$ and $n=3$.

By using the above model of nonlinear regression analysis, we can obtain different nonlinear models for the domination topological indicesas follows:

$$
\begin{gathered}
\log (\text { M.P. })=5.2-0.005 \log D M_{1}, \\
\log (\text { M.P. })=5.2-0.0053 \log D M_{2}, \\
\log (\text { M.P. })=5.2-0.01 \log D M_{1}^{*}, \\
\log (\text { M.P. })=5.2-0.004 \log D F, \\
\log \text { (M.P. })=5.2-0.005 \log D H, \\
\log (\text { M.P. })=5.2-0.0052 \log D F^{*} .
\end{gathered}
$$

Now, the predicted values of physiochemical property are given in Table 5. 
TABLE 5. The values of M.P. Predicted by domination topological indices

\begin{tabular}{|c|c|c|c|c|c|c|}
\hline \multirow{2}{*}{$\begin{array}{c}\text { Polymer } \\
\text { name }\end{array}$} & \multicolumn{7}{|c|}{ M.P. - Predicted by } \\
\cline { 2 - 7 } & $D M_{1}$ & $D M_{2}$ & $D M_{1}^{*}$ & $D F$ & $D H$ & $D F^{*}$ \\
\hline \hline PHB[1] & 175.3 & 175.1 & 172.1378 & 175.1 & 174.2 & 174.6 \\
\hline PHV[1] & 173.9 & 173.5 & 170.537 & 173.4 & 172.8 & 173.1 \\
\hline PHBV[1] & 168.7 & 168.03 & 164.882 & 167.4 & 167.58 & 167.7 \\
\hline PHB[2] & 169.9 & 169.3 & 166.2931 & 168.88 & 168.86 & 168.9 \\
\hline PHV[2] & 167.5 & 166.7 & 163.561 & 165.94 & 166.3 & 166.3 \\
\hline PHBV[2] & 157.9 & 156.7 & 153.9177 & 154.9 & 156.9 & 156.6 \\
\hline PHB[3] & 165.01 & 164.1 & 161.0975 & 163.11 & 163.9 & 163.8 \\
\hline PHV[3] & 161.4 & 160.3 & 157.3411 & 158.9 & 160.3 & 160.2 \\
\hline PHBV[3] & 148.11 & 146.3 & 144.0490 & 143.5 & 147.2 & 146.5 \\
\hline
\end{tabular}

Figure 4 indicates how much the predicted values of physio-chemical properties are correlated with the wellknown physio-chemical properties. The degree of correlation between any two data sets is measured by the correlation coefficient $(R)$. When the value of $R$ becomes close to unity, two data sets are more correlated. The QSPR study of domination indices reveals that these domination indices can be helpful in predicting the Melting Point (M.P.). From Fig. 4, the range of the correlation is $0.56<R<0.57$ which shows a good correlation of predicted values of Melting Point (M.P.) with exact values of M.P. In fact, these obtained values for the correlation coefficient for these domination indices are satisfactory. On another hand, all domination indices are good to predict the M.P. of these polymers. Melting Point is an important physicochemical property using these domination indices to predict the values of this property is very useful and saves time and money. It has been shown that these indices can be considered useful molecular descriptors in QSPR research of polymers.

The correlation coefficient values of predicted physicochemical properties with the exact values of physio-chemical properties of the chemical compounds used in this study are given in Table 6.
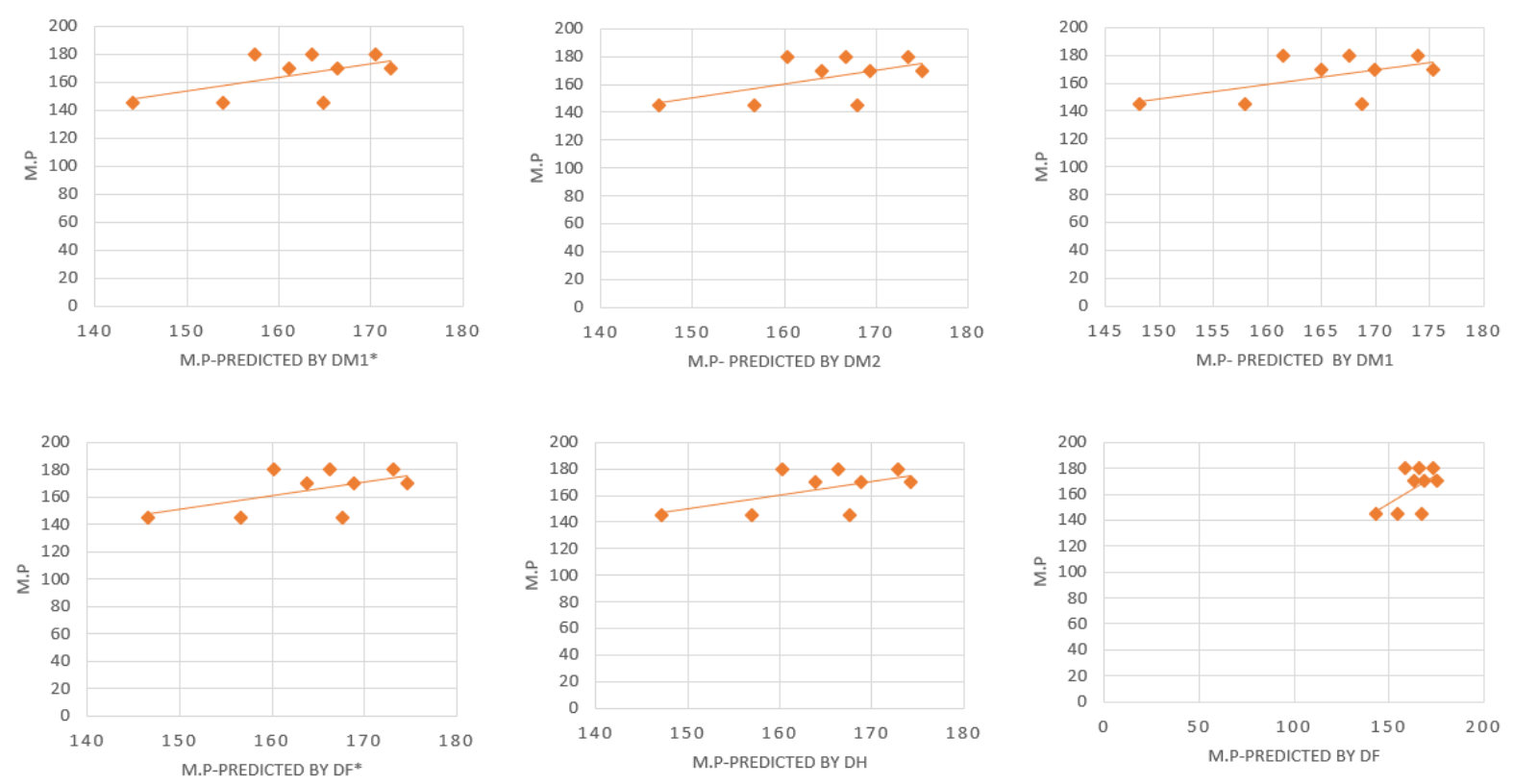

FIG. 4. Graphical relationships between predicted values of M.P., and the exact values of M.P. 
TABLE 6. The correlation coefficient values of predicted physicochemical properties with the exact values of physio-chemical properties

\begin{tabular}{|c|c|c|c|c|c|c|}
\hline \multirow{2}{*}{$\begin{array}{c}\text { Physicochemical } \\
\text { property }\end{array}$} & \multicolumn{6}{|c|}{ M.P. - Predicted by } \\
\cline { 2 - 7 } & $D M_{1}$ & $D M_{2}$ & $D M_{1}^{*}$ & $D F$ & $D H$ & $D F^{*}$ \\
\hline \hline M.P. & 0.57 & 0.562 & 0.56 & 0.563 & 0.562 & 0.562 \\
\hline
\end{tabular}

\section{Conclusion}

We calculated domination topological indices for PHB, PHV, and their copolymer, PHBV. There are many different applications of these polymers that resemble petroleum-based plastic such as polypropylene, which is useful in kinking many of the physical and chemical properties of these polymers with domination topological indices. We have also discussed the QSPR analysis of PHB, PHV, and their copolymer, PHBV. The cases in which good correlations were obtained suggested the validity of the calculated topological indices to be further used to predict the physicochemical properties of chemical compounds.

\section{Acknowledgements}

The authors would like to thank the reviewer for his constructive suggestions and useful comments which resulted in an improved version of this paper.

\section{References}

[1] Lemoigne M. Produits de deshydration et de polymerisation de lacide-oxybutyrique. Bull. Soc. Chim. Biol., 1926, 8, P. 770-782.

[2] Shrivastav A., Kim H.Y., Kim Y.R. Advances in the applications of polyhydroxyalkanoate nanoparticles for novel drug delivery system. Biomed. Res. Int., 2013, 12, P. 1-12.

[3] Hazer B. Amphiphilic poly(3-hydroxy alkanoate)s: potential candidates for medical applications. Int. J. of Polymer Science, $2010,423460$.

[4] Lau N.S., Sudesh K. Revelation of the ability of Burkholderi a sp, USM (JCM 15050) PHAs syn-thase to polymerize 4-hydroxybutyrate monomer. AMB Express, 2012, 2 (1), 41.

[5] Brigham C.J., Bhubalan K., et al. Characterization of the highly active polyhydroxyalkanoate synthase of Chromobacterium sp.strain USM2. Appl. Environ. Microbiol., 2011, 77 (9), P. 2926-2933.

[6] Budde C.F., Riedel S.L., et al. Growth and polyhydroxybutyrate production by Ralstonia eutropha in emulsi ed plant oil medium. Appl. Microbiol. Biotechnol., 2011, 89 (5), P. 1611-1619.

[7] Sudesh K., Abe H., Doi Y. Synthesis, structure and properties of polyhydroxyalkanoates: biological polyesters. Progress in Polymer Science, 2000, 25, P. 1503-1555.

[8] François J., Couturier A., Letourneur R., Liedloff M. On the number of minimal dominating sets on some graph classes. Theor. Computer Science, 2015, 562, P. 634-642.

[9] Akhter S., Imran M., Farahani M.R., Javaid I. On topological properties of hexagonal and silicate networks. Hacettepe J. of Mathematics and Statistics, 2019, 48 (3), P. 711-723.

[10] Ahmed H., Alwardi A., Salestina R.M. Domination Topological Indices and Polynomial of Some Chemical Structures Applied for the Treatment of COVID-19 Patients. Biointerface Research in Applide Chemistry, 2021, 5, P. 13290-13302.

[11] Ahmed H., Farahani M.R., Alwardi A., Salestina R.M. Domination topological properties of some chemical structures using polynomial approach. Eurasian Chemical Communications, 2021, 3 (4), P. 210-218.

[12] Ahmed H., Alwardi A., Salestina R.M. Domination topological indices and their polynomials of a firefly graph. J. of Discrete Mathematical Sciences and Cryptography, 2021, 24 (2) P. 325-341.

[13] Wazzan S., Saleh A. On the First and Second Locating Zagreb Indices of Graphs. Applied Mathematics, 2019,10 (10), P. 805-816.

[14] Wazzan S., Saleh A. Locating and Multiplicative Locating Indices of Graphs with QSPR Analysis. J. of Mathematics, $2021,5516321$.

[15] Hanan Ahmed A.M., Alwardi A., Salestina R.M. On Domination Topological Indices of Graphs. Int. J. Anal. Appl., 2021,19 (1), P. $47-64$.

[16] Ahmed H., Alwardi A., Salestina R.M., Soner N.D. Forgotten domination, hyper domination and modified forgotten domination indices of graphs. J. of Discrete Mathematical Sciences and Cryptography, 2021, 24 (2), P. 353-368.

[17] Lichtenthaler F.W. Carbohydrates as Organic Raw Materials. Ullmann's Encyclopedia of Industrial Chemistry, 2010, Wiley-VCH Verlag $\mathrm{GmbH} \& \mathrm{Co}$. KGaA. 